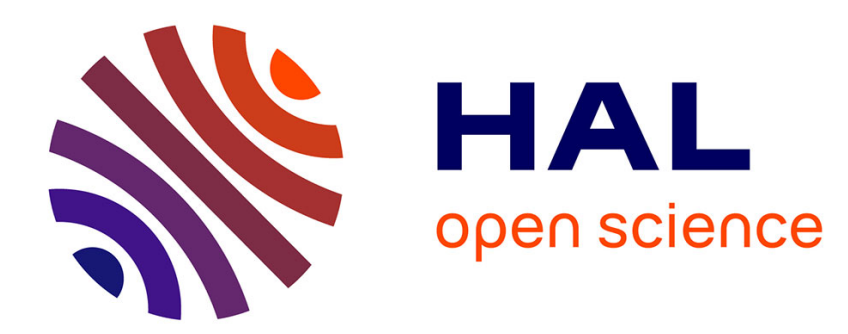

\title{
TIME FRACTIONAL SCHRÖDINGER EQUATION
}

Arnaud Rougirel, Hassan Emamirad

\section{To cite this version:}

Arnaud Rougirel, Hassan Emamirad. TIME FRACTIONAL SCHRÖDINGER EQUATION. 2018. hal-01794446

\author{
HAL Id: hal-01794446 \\ https://hal.science/hal-01794446 \\ Preprint submitted on 17 May 2018
}

HAL is a multi-disciplinary open access archive for the deposit and dissemination of scientific research documents, whether they are published or not. The documents may come from teaching and research institutions in France or abroad, or from public or private research centers.
L'archive ouverte pluridisciplinaire HAL, est destinée au dépôt et à la diffusion de documents scientifiques de niveau recherche, publiés ou non, émanant des établissements d'enseignement et de recherche français ou étrangers, des laboratoires publics ou privés. 


\title{
TIME FRACTIONAL SCHRÖDINGER EQUATION
}

\author{
HASSAN EMAMIRAD AND ARNAUD ROUGIREL
}

\begin{abstract}
We propose a time fractional extension of the Schrödinger equation that keeps the main mechanical and quantum properties of the classical Schrödinger equation. This extension is shown to be equivalent to another well identified time first order PDE with fractional hamiltonian.
\end{abstract}

\section{INTRODUCTION}

We would like to address the issue of what are suitable time fractional extensions of the Schrödinger equation. Our approach consists in, first, selecting some (important) properties of the Schrödinger equation; secondly, finding a time fractional equation which conserves these properties.

We have selected two properties: (i) the conservation of the $L^{2}$-norm of wave functions. That point is central w.r.t. the probabilistic character of quantum mechanics objects. (ii) The dynamics of the Schrödinger equation, i.e. the time reversibility of solutions which is related to the fact that the related solution operator is a group. Indeed, in any reasonable mechanical theory, trajectories of autonomous systems must be described by equations generating a group.

It is clear that the use of time fractional operators as $\mathbf{D}_{0, t}^{\alpha}$, do not allow to match the above requirements. We refer to Remark 4.1 below for details, and to [Die10] or [SKM93] for more information on fractional derivatives. Regarding the dynamics of time fractional equations, we refer to [ER17].

In this paper, the real number $\alpha$ is always supposed to range between 0 and 1 .

In order to recover the above properties, we consider time fractional operators with lower bound $-\infty$, i.e. $\mathbf{D}_{-\infty, t}^{\alpha}$. More precisely, if, for simplicity, we restrict our attention to the Hamiltonian $\hat{H}:=-\Delta$, then we will show that the problem

$$
\mathbf{D}_{-\infty, t}^{\alpha} u=-\mathrm{i}^{\alpha} \Delta u, \quad u(0)=v,
$$

admits a unique solution $u$ in some suitable function space. Theorem 4.1 states that the solution to (1.1) is given by

$$
u(t)=\mathrm{e}^{\mathrm{i} t(-\Delta)^{1 / \alpha}} v,
$$

hence the $L^{2}$-norm of $u$ is conserved and the solution operator of (1.1) is a group. Then, according to Corollary 4.3, (1.1) is equivalent to

$$
\frac{\mathrm{d}}{\mathrm{d} t} u=\mathrm{i}(-\Delta)^{1 / \alpha} u, \quad u(0)=v .
$$

The coefficient $\mathrm{i}^{\alpha}$ in (1.1) is not surprising since we know from the work of Naber, in [Nab04], that the operator $\mathbf{D}_{0, t}+\mathrm{i} \Delta$ has a parabolic behavior (see Remark 4.1).

Date: May 17, 2018.

This research was in part supported by a grant from IPM \# 91470221. 
In this paper, we consider more generally abstract time fractional Schrödinger equations of the form

$$
\frac{\mathrm{d}}{\mathrm{d} t} u=\mathrm{i}^{\alpha} A u
$$

where $A$ is a positive self-adjoint operator.

The outline is as follows. The forcoming section is dedicated to preliminaries regarding fractional derivatives, in particular time fractional weak derivatives. In section 3, we solve (1.3) in the case where the underlying Hilbert space in $\mathbb{R}$. That turns out to be the corner stone of our work since, in section 4, we solve (1.3) by diagonalisation using spectral theory.

\section{Fractional Derivatives With LOWER BOUnd $-\infty$}

We start with the convolution of functions defined on $\mathbb{R}$, with the fractional kernel, whose definition is as follow.

Definition 2.1. For $\beta \in(0, \infty)$, let us denote by $g_{\beta}$ the function of $L_{l o c}^{1}([0, \infty))$ defined for a.e. $t>0$ by

$$
g_{\beta}(t)=\frac{1}{\Gamma(\beta)} t^{\beta-1} .
$$

Let $X$ be a complex Banach space with norm $\|\cdot\|$.

Proposition 2.1. Let $\alpha \in(0,1)$ and $u \in L^{1}(\mathbb{R} ; X) \cap L^{\infty}(\mathbb{R} ; X)$. Then, for all $t \in \mathbb{R}$, the function

is integrable on $(-\infty, t)$ and

$$
(-\infty, t) \rightarrow X, \quad y \mapsto g_{\alpha}(t-y) u(y)
$$

$$
\sup _{t \in \mathbb{R}} \int_{-\infty}^{t} g_{\alpha}(t-y)\|u(y)\| \mathrm{d} y<\infty .
$$

Proof. For all $t \in \mathbb{R}$, we have

$$
\begin{aligned}
\int_{-\infty}^{t} g_{\alpha}(t-y)\|u(y)\| \mathrm{d} y & \leq \int_{-\infty}^{t-1} g_{\alpha}(t-y)\|u(y)\| \mathrm{d} y+\int_{t-1}^{t} g_{\alpha}(t-y)\|u(y)\| \mathrm{d} y \\
& \leq g_{\alpha}(1)\|u\|_{L^{1}(\mathbb{R} ; X)}+g_{\alpha+1}(1)\|u\|_{L^{\infty}(\mathbb{R} ; X)} .
\end{aligned}
$$

Under the assumptions and notation of Proposition 2.1, we put, for all $t \in \mathbb{R}$,

$$
g_{\alpha} * u(t):=\int_{-\infty}^{t} g_{\alpha}(t-y) u(y) \mathrm{d} y=\int_{0}^{\infty} g_{\alpha}(y) u(t-y) \mathrm{d} y .
$$

Also, we define

$$
g_{\alpha} *^{\prime} u(t):=\int_{t}^{\infty} g_{\alpha}(y-t) u(y) \mathrm{d} y=\int_{0}^{\infty} g_{\alpha}(y) u(t+y) \mathrm{d} y .
$$

In some sense, these convolutions are adjoints. More precisely, we have the following result, whose easy proof is left to the reader.

Proposition 2.2. Let $\alpha \in(0,1), u \in L^{1}(\mathbb{R} ; X) \cap L^{\infty}(\mathbb{R} ; X)$ and $\psi \in L^{1}(\mathbb{R}) \cap L^{\infty}(\mathbb{R})$. Then

$$
\int_{\mathbb{R}} g_{\alpha} * u(t) \psi(t) \mathrm{d} t=\int_{\mathbb{R}} u(t) g_{\alpha} *^{\prime} \psi(t) \mathrm{d} t .
$$


Then we may give the following definition of fractional derivatives.

Definition 2.2. Let $\alpha \in(0,1)$ and $u \in L^{1}(\mathbb{R} ; X) \cap L^{\infty}(\mathbb{R} ; X)$. We say that $u$ admits a (forward) derivative of order $\alpha$ in $L^{\infty}(\mathbb{R} ; X)$ if

$$
g_{1-\alpha} * u \in W^{1, \infty}(\mathbb{R} ; X) .
$$

In this case, its (forward) derivative of order $\alpha$ is the function of $L^{\infty}(\mathbb{R} ; X)$ defined by

$$
\mathbf{D}_{-\infty, t}^{\alpha} u:=\frac{\mathrm{d}}{\mathrm{d} t}\left\{g_{1-\alpha} * u\right\} .
$$

Definition 2.3. Let $\alpha \in(0,1)$ and $u \in W^{1,1}(\mathbb{R} ; X) \cap W^{1, \infty}(\mathbb{R} ; X)$. Then we say that $u$ admits a backward derivative of order $\alpha$ in $L^{\infty}(\mathbb{R} ; X)$ if

$$
g_{1-\alpha} *^{\prime} \frac{\mathrm{d}}{\mathrm{d} t} u \in L^{\infty}(\mathbb{R} ; X) .
$$

In this case, its backward derivative of order $\alpha$ is the function of $L^{\infty}(\mathbb{R} ; X)$ defined by

$$
\mathbf{D}_{t, \infty}^{\alpha} u:=g_{1-\alpha} *^{\prime} \frac{\mathrm{d}}{\mathrm{d} t} u \text {. }
$$

Let $\mathcal{S}(\mathbb{R})$ denote the Schwartz space of rapidly decreasing complex functions defined on $\mathbb{R}$.

Proposition 2.3. Let $\alpha \in(0,1), u \in L^{1}(\mathbb{R} ; X) \cap L^{\infty}(\mathbb{R} ; X)$ and $\varphi \in \mathcal{S}(\mathbb{R})$. Assume that $u$ admits a fractional derivative of order $\alpha$ in $L^{\infty}(\mathbb{R} ; X)$. Then

$$
\int_{\mathbb{R}} \mathbf{D}_{-\infty, t}^{\alpha} u(t) \varphi(t) \mathrm{d} t=-\int_{\mathbb{R}} u(t) \mathbf{D}_{t, \infty}^{\alpha} \varphi(t) \mathrm{d} t .
$$

Proof. Integrate by parts and use Proposition 2.2 .

We will now introduce fractional derivatives in the sense of distributions. The following result makes possible such a definition.

Proposition 2.4. Let $\alpha \in(0,1)$ and $\varphi \in \mathcal{S}(\mathbb{R})$. Then $\mathbf{D}_{t, \infty}^{\alpha} \varphi \in L^{1}(\mathbb{R})$ and

$$
\left\|\mathbf{D}_{t, \infty}^{\alpha} \varphi\right\|_{L^{1}(\mathbb{R})} \leq C\left(\|\varphi\|_{L^{1}(\mathbb{R})}+\left\|\varphi^{\prime}\right\|_{L^{1}(\mathbb{R})}\right),
$$

where $\varphi^{\prime}:=\frac{\mathrm{d}}{\mathrm{d} t} \varphi$ and the constant $C$ depends only on $\alpha$.

Proof. For any fixed time $t$ in $\mathbb{R}$, let $v(y):=\varphi(y+t)-\varphi(t)$. Integrating by part, we get

Hence

$$
\alpha \int_{0}^{\infty} y^{-\alpha-1} v(y) \mathrm{d} y=\int_{0}^{\infty} y^{-\alpha} \varphi^{\prime}(y+t) \mathrm{d} y .
$$

$$
\mathbf{D}_{t, \infty}^{\alpha} \varphi(t)=\frac{1}{|\Gamma(-\alpha)|} \int_{0}^{\infty} y^{-\alpha-1}(\varphi(y+t)-\varphi(t)) \mathrm{d} y,
$$

and

$$
\begin{aligned}
& \left|\Gamma(-\alpha) \mathbf{D}_{t, \infty}^{\alpha} \varphi(t)\right| \leq \int_{0}^{1} y^{-\alpha}\left|\frac{\varphi(y+t)-\varphi(t)}{y}\right| \mathrm{d} y \quad+\int_{1}^{\infty} y^{-\alpha-1}|\varphi(y+t)-\varphi(t)| \mathrm{d} y \\
& =: \quad I_{1}(t) \quad+\quad I_{2}(t),
\end{aligned}
$$

with obvious notation for $I_{1}(t)$ and $I_{2}(t)$. 
Let us show that $I_{1}$ is integrable on $\mathbb{R}$. For, since

$$
\frac{\varphi(y+t)-\varphi(t)}{y}=\int_{0}^{1} \varphi^{\prime}(y s+t) \mathrm{d} s, \quad \forall y \in(0,1]
$$

we derive

$$
\int_{\mathbb{R}}\left|I_{1}(t)\right| \mathrm{d} t \leq \int_{0}^{1} y^{-\alpha} \mathrm{d} y \int_{0}^{1} \mathrm{~d} s \int_{\mathbb{R}}\left|\varphi^{\prime}(y s+t)\right| \mathrm{d} t=\frac{\left\|\varphi^{\prime}\right\|_{L^{1}(\mathbb{R})}}{1-\alpha} .
$$

Regarding $I_{2}(t)$, we have

$$
\begin{aligned}
\int_{\mathbb{R}}\left|I_{2}(t)\right| \mathrm{d} t & \leq \int_{1}^{\infty} y^{-\alpha-1} \int_{\mathbb{R}}|\varphi(y+t)|+|\varphi(t)| \mathrm{d} t \\
& =\frac{2}{\alpha}\|\varphi\|_{L^{1}(\mathbb{R})} .
\end{aligned}
$$

That estimate allows us to define fractional derivatives in the sense of distributions. Indeed, (2.3) shows that, for each $u \in L^{\infty}(\mathbb{R} ; X)$, the linear map

$$
\mathcal{S}(\mathbb{R}) \rightarrow X, \quad \varphi \mapsto-\int_{\mathbb{R}} u(t) \mathbf{D}_{t, \infty}^{\alpha} \varphi(t) \mathrm{d} t
$$

is a tempered distribution. The set of tempered distributions with values in $X$ is denoted by $\mathcal{S}^{\prime}(\mathbb{R} ; X)$. That allows us to set the following definition.

Definition 2.4. Let $\alpha \in(0,1), X$ be a complex Banach space and $u \in L^{\infty}(\mathbb{R} ; X)$. Then the weak derivative of $u$ is the $X$-valued tempered distribution, denoted by $\mathbf{D}_{-\infty, t}^{\alpha} u$, and defined, for all $\varphi \in \mathcal{S}(\mathbb{R})$, by

$$
\left\langle\mathbf{D}_{-\infty, t}^{\alpha} u, \varphi\right\rangle=-\int_{\mathbb{R}} u(t) \mathbf{D}_{t, \infty}^{\alpha} \varphi(t) \mathrm{d} t .
$$

If we want to highlight the duality taking place in the above bracket, we will write

$$
\left\langle\mathbf{D}_{-\infty, t}^{\alpha} u, \varphi\right\rangle_{\mathcal{S}^{\prime}(\mathbb{R} ; X), \mathcal{S}(\mathbb{R})} \quad \text { or } \quad\left\langle\mathbf{D}_{-\infty, t}^{\alpha} u, \varphi\right\rangle_{\mathcal{S}^{\prime}(\mathbb{R} ; X)}
$$

instead of $\left\langle\mathbf{D}_{-\infty, t}^{\alpha} u, \varphi\right\rangle$.

In this paper, we will need to compute $\mathbf{D}_{-\infty, t}^{\alpha} u$ for bounded functions $u$. For such functions, the integral

$$
\int_{-\infty}^{t} g_{1-\alpha}(t-y) u(y) \mathrm{d} y
$$

is, in general, not absolutely convergent. That point turns out to be a major drawback; and we will explain the reason in the sequel.

Works on fractional calculus with lower terminal $-\infty$ and not absolutely convergent integrals, go back, at least to 1938 with the smart paper [Lov38]. The difficulties when dealing with non absolutely convergent integral are illustrated by Theorem 5 in [Lov38], which states, roughly speaking that each $u \in L^{\infty}(\mathbb{R})$ such that

$$
\sup _{t \in \mathbb{R}}\left|\int_{0}^{t} u(y) \mathrm{d} y\right|<\infty
$$

satisfies, for all $t \in \mathbb{R}$, the following (hard to prove) identity

$$
g_{1-\alpha} *\left(g_{\alpha} * u\right)(t)=g_{1-\alpha} *\left(g_{\alpha} * u\right)(0)+\int_{0}^{t} u(y) \mathrm{d} y .
$$


That result is quiet surprising since, extending $g_{\alpha}$ by 0 outside of $(0, \infty)$, we have

$$
g_{1-\alpha} * g_{\alpha}(t)=\left\{\begin{array}{ll}
1 & \text { if } t>0 \\
0 & \text { if } t<0
\end{array} ;\right.
$$

so that the convolution

$$
\left(g_{1-\alpha} * g_{\alpha}\right) * u(t)=\int_{-\infty}^{t} u(y) \mathrm{d} y
$$

is not defined in general.

In this paper, we deal with bounded functions satisfying typically (2.4). However, Love's approach (see also [BMRST16]) allowed us to obtain only partial results. That is, we must assume that $\alpha=1 / n$ for some positive integer $n$.

\section{Time FRACTIONAL EQUATIONS IN $\mathbb{R}$}

We will use the framework of Section 2 with $X=\mathbb{C}$. For $a \in \mathbb{R}$ and $\alpha \in(0,1)$, we consider this equation:

$$
\left\{\begin{array}{l}
\text { Find } u \in L^{\infty}(\mathbb{R}) \quad \text { such that } \\
\mathbf{D}_{-\infty, t}^{\alpha} u=(\mathrm{i} a)^{\alpha} u, \quad \text { in } \mathcal{S}^{\prime}(\mathbb{R}) .
\end{array}\right.
$$

Of course, in (3.1), $\mathbf{D}_{-\infty, t}^{\alpha} u$ is understood in the sense of Definition 2.4.

For $\psi \in L^{2}\left(\mathbb{R}^{d}\right)$, we denote by $\mathcal{F} \psi$ or $\hat{\psi}$ its Fourier transform. More precisely, for $\varphi \in L^{1}(\mathbb{R})$, we have

$$
\hat{\varphi}(\sigma)=c_{\mathcal{F}} \int_{\mathbb{R}} \mathrm{e}^{-\mathrm{i} \sigma t} \varphi(t) \mathrm{d} t, \quad \mathcal{F}^{-1} \varphi(t)=c_{\mathcal{F}^{-1}} \int_{\mathbb{R}} \mathrm{e}^{\mathrm{i} t \sigma} \varphi(\sigma) \mathrm{d} \sigma,
$$

where $c_{\mathcal{F}}, c_{\mathcal{F}^{-1}}$ denote reader's favorite constants of normalization, whose product equals $\frac{1}{2 \pi}$.

Remark 3.1. Solving the fractional equation

$$
\mathbf{D}_{-\infty, t}^{\alpha} u=(\mathrm{i} a)^{\alpha} u
$$

is a touchy business.

First, it is known that the function $u_{1}: t \mapsto \exp (\mathrm{i} a t)$ satisfies

$$
\int_{0}^{\infty} g_{1-\alpha}(y) u_{1}(t-y) \mathrm{d} y=(\mathrm{i} a)^{\alpha-1} u_{1}(t) .
$$

Thus, $u_{1}$ solves (3.3) in some "classical sense". However, the above integral is not absolutely convergent. Thus it is not clear how to get uniqueness for (3.3) in a space containing $u_{1}$ (typically $C_{\mathrm{b}}(\mathbb{R})$ ). This is the reason why we use time fractional derivatives in the sense of distributions to solve (3.3).

But then, existence for (3.1) is tricky since, we have not been able to show directly that $u_{1}$ solves (3.1). Indeed, Fubini's Theorem being not applicable, the path from

to

$$
\int_{\mathbb{R}} \mathrm{e}^{\mathrm{i} a t} \mathbf{D}_{t, \infty}^{\alpha} \varphi(t) \mathrm{d} t=\int_{\mathbb{R}} \mathrm{e}^{\mathrm{i} a t} \int_{t}^{\infty} g_{1-\alpha}(y-t) \varphi^{\prime}(y) \mathrm{d} y
$$

$$
\int_{\mathbb{R}} \varphi^{\prime}(y) \mathrm{d} y \int_{-\infty}^{y} \mathrm{e}^{\mathrm{i} a t} g_{1-\alpha}(y-t) \mathrm{d} t
$$

is not clear. We overcome this difficulty by using Fourier transforms. 
The last difficulty is technical and concerns the use of Fourier transforms. More precisely, starting from (3.3), we obtain formally by Fourier transform,

$$
(\mathrm{i} \sigma)^{\alpha} \hat{u}=(\mathrm{i} a)^{\alpha} \hat{u} .
$$

However, if $u=u_{1}$ then

$$
(\mathrm{i} \sigma)^{\alpha} \hat{u}=\frac{1}{c_{\mathcal{F}-1}}(\mathrm{i} \sigma)^{\alpha} \delta_{a}
$$

which has no meaning since the function $\sigma \mapsto(\mathrm{i} \sigma)^{\alpha}$, being not smooth at $\sigma=0$, cannot multiply $\delta_{a}$, the Dirac mass at the point $a$.

In order to overcome this difficulty, we first determine the support of $\hat{u}$, which turns out to be discrete, as expected; and then compute $u$ by inverse Fourier transform.

Theorem 3.1. Let $a \in \mathbb{R}, \alpha \in(0,1)$ and $u \in L^{\infty}(\mathbb{R})$. Then $u$ is a solution of (3.1) iff there exists $k \in \mathbb{C}$ such that

$$
u(t)=k \exp (\mathrm{i} a t), \quad \text { a.e. } \quad t \in \mathbb{R} .
$$

The following lemma will be usefull in the proof of Theorem 3.1.

Lemma 3.2. Let $\varphi \in \mathcal{S}(\mathbb{R})$. Then, for all $\sigma \in \mathbb{R}$,

$$
\begin{aligned}
\mathcal{F}\left(\mathbf{D}_{-\infty, t}^{\alpha} \varphi\right)(\sigma) & =(\mathrm{i} \sigma)^{\alpha} \hat{\varphi}(\sigma) \\
\mathbf{D}_{\sigma, \infty}^{\alpha} \hat{\varphi}(\sigma) & =-\mathcal{F}\left((\mathrm{i} t)^{\alpha} \varphi\right)(\sigma) .
\end{aligned}
$$

Proof. For the proof of (3.4), we refer to [SKM93, Section 7.1]. We establish (3.5) by a duality argument. Indeed, for all $\varphi$ and $\psi$ in $\mathcal{S}(\mathbb{R})$, there holds, by Proposition 2.3

$$
\begin{aligned}
\int_{\mathbb{R}} \mathbf{D}_{\sigma, \infty}^{\alpha} \hat{\varphi}(\sigma) \psi(\sigma) \mathrm{d} \sigma & =-\int_{\mathbb{R}} \hat{\varphi}(\sigma) \mathbf{D}_{-\infty, \sigma}^{\alpha} \psi(\sigma) \mathrm{d} \sigma \\
& =-\int_{\mathbb{R}} \varphi(\sigma) \mathcal{F}\left(\mathbf{D}_{-\infty, t}^{\alpha} \psi\right)(\sigma) \mathrm{d} \sigma
\end{aligned}
$$

by Plancherel's formula. Thus with (3.4) and Plancherel's formula once again, we get

$$
\begin{aligned}
\int_{\mathbb{R}} \mathbf{D}_{\sigma, \infty}^{\alpha} \hat{\varphi}(\sigma) \psi(\sigma) \mathrm{d} \sigma & =-\int_{\mathbb{R}} \varphi(\sigma)(\mathrm{i} \sigma)^{\alpha} \hat{\psi}(\sigma) \mathrm{d} \sigma \\
& =-\int_{\mathbb{R}} \mathcal{F}\left((\mathrm{i} t)^{\alpha} \varphi\right)(\sigma) \psi(\sigma) \mathrm{d} \sigma
\end{aligned}
$$

That proves (3.5).

Proof of Theorem 3.1. (i) Let us show that the function $u: t \mapsto \mathrm{e}^{\mathrm{i} a t}$ is solution to (3.1). For, we compute

$$
\begin{aligned}
\left\langle\mathcal{F}\left(\mathbf{D}_{-\infty, t}^{\alpha} u\right), \varphi\right\rangle_{\mathcal{S}^{\prime}(\mathbb{R}), \mathcal{S}(\mathbb{R})} & =\left\langle\mathbf{D}_{-\infty, t}^{\alpha} u, \hat{\varphi}\right\rangle \\
& =-\int_{\mathbb{R}} u(\sigma) \mathbf{D}_{\sigma, \infty}^{\alpha} \hat{\varphi}(\sigma) \mathrm{d} \sigma \\
& =\int_{\mathbb{R}} \mathrm{e}^{\mathrm{i} a \sigma} \mathcal{F}\left((\mathrm{i} t)^{\alpha} \varphi\right)(\sigma) \mathrm{d} \sigma
\end{aligned}
$$


by (3.5). Besides, the Fourier transform of $t \mapsto(\mathrm{i} t)^{\alpha} \varphi(t)$ belongs to $L^{1}(\mathbb{R})$ by $(3.5)$ and Proposition 2.4. Then the latter integral is equal, by inverse Fourier transform (see $(3.2)$ ), to

$$
\begin{aligned}
\frac{1}{c_{\mathcal{F}^{-1}}}(\mathrm{i} a)^{\alpha} \varphi(a)=(\mathrm{i} a)^{\alpha}\left\langle\frac{\delta_{a}}{c_{\mathcal{F}^{-1}}}, \varphi\right\rangle & =(\mathrm{i} a)^{\alpha}\left\langle\mathcal{F}\left(\mathrm{e}^{\mathrm{i} a t}\right), \varphi\right\rangle \\
& =(\mathrm{i} a)^{\alpha}\langle\mathcal{F} u, \varphi\rangle .
\end{aligned}
$$

Thus by invertibility of the Fourier transform in $\mathcal{S}^{\prime}(\mathbb{R})$, we deduce that $t \mapsto \mathrm{e}^{\mathrm{i} a t}$ is solution to (3.1).

(ii) Conversely, let us show that each solution $u$ to (3.1) is proportional to some exponential function. Computing as above, we get, for each test-function $\varphi$ in $\mathcal{S}(\mathbb{R})$,

$$
\left\langle\mathcal{F}\left(\mathbf{D}_{-\infty, t}^{\alpha} u\right), \varphi\right\rangle_{\mathcal{S}^{\prime}(\mathbb{R}), \mathcal{S}(\mathbb{R})}=\int_{\mathbb{R}} u(\sigma) \mathcal{F}\left((\mathrm{i} t)^{\alpha} \varphi\right)(\sigma) \mathrm{d} \sigma .
$$

Thus, since $u$ solves (3.1),

$$
0=\int_{\mathbb{R}} u(\sigma) \mathcal{F}\left(\left\{(\mathrm{i} t)^{\alpha}-(\mathrm{i} a)^{\alpha}\right\} \varphi\right)(\sigma) \mathrm{d} \sigma .
$$

Now, set

$$
I_{a}:=\left\{\begin{array}{ll}
\{0\} & \text { if } a=0 \\
\{0, a\} & \text { if } a \neq 0
\end{array} .\right.
$$

Then, for each $\psi \in \mathcal{S}(\mathbb{R})$ with support, denoted by $\operatorname{supp} \psi$, in $\mathbb{R} \backslash I_{a}$, we may find a function $\varphi \in \mathcal{S}(\mathbb{R})$ so that

$$
\psi(t)=\left((\mathrm{i} t)^{\alpha}-(\mathrm{i} a)^{\alpha}\right) \varphi(t), \quad \forall t \in \operatorname{supp} \psi .
$$

There results that

$$
\langle\hat{u}, \psi\rangle_{\mathcal{S}^{\prime}(\mathbb{R}), \mathcal{S}(\mathbb{R})}=0 .
$$

Thus the support of the distribution $\hat{u}$ lies in $I_{a}$. By standard results in the theory of distributions, we infer that there exist some integer $n \geq 0$ and $c_{k}, d_{k} \in \mathbb{C}(k=$ $0, \ldots, n)$ such that

$$
\hat{u}=\sum_{k=0}^{n} c_{k} \delta_{0}^{(k)}+d_{k} \delta_{a}^{(k)},
$$

where $\delta_{a}$ denotes the Dirac mass at $a$. Hence, for other constants still labeled $c_{k}$ and $d_{k}$

$$
u(t)=\sum_{k=0}^{n} c_{k} t^{k}+d_{k} t^{k} \mathrm{e}^{\mathrm{i} a t}, \quad \forall t \in \mathbb{R} .
$$

Since $u$ is bounded, $u(t)=c_{0}+d_{0} \mathrm{e}^{\mathrm{i} a t}$. Finally, according to the existence part (i) of this proof, we have

$$
\mathbf{D}_{-\infty, t}^{\alpha}\left(c_{0}+d_{0} \mathrm{e}^{\mathrm{i} a t}\right)=d_{0}(\mathrm{i} a)^{\alpha} \mathrm{e}^{\mathrm{i} a t} \quad \text { in } \mathcal{S}^{\prime}(\mathbb{R}) .
$$

On the other hand, since $u$ solves (3.1), there holds

$$
\mathbf{D}_{-\infty, t}^{\alpha}\left(c_{0}+d_{0} \mathrm{e}^{\mathrm{i} a t}\right)=(\mathrm{i} a)^{\alpha}\left(c_{0}+d_{0} \mathrm{e}^{\mathrm{i} a t}\right) .
$$

Thus, for all $t \in \mathbb{R}$, we have $u(t)=d_{0} \exp (\mathrm{i} a t)$. 


\section{TIME FRACTIONAL SChrÖDINGER EQUATION}

Let $\alpha \in(0,1), d \geq 1$ be an integer and $A: D(A) \subseteq L^{2}\left(\mathbb{R}^{d}\right) \rightarrow L^{2}\left(\mathbb{R}^{d}\right)$ be a unbounded operator on $L^{2}\left(\mathbb{R}^{d}\right)$ with domain $D(A)$. For each $v \in D(A)$, we consider the following Time Fractional Schrödinger Equation

$$
\left\{\begin{array}{l}
\text { Find } u \in C_{\mathrm{b}}(\mathbb{R}, D(A)) \text { such that } \\
\mathbf{D}_{-\infty, t}^{\alpha} u=\mathrm{i}^{\alpha} A u, \quad \text { in } \mathcal{S}^{\prime}\left(\mathbb{R}, L^{2}\left(\mathbb{R}^{d}\right)\right) \\
u(0)=v .
\end{array}\right.
$$

In (4.1), $C_{\mathrm{b}}(\mathbb{R}, D(A))$ is the space of bounded and continuous functions defined on $\mathbb{R}$ with values in $D(A)$.

Remark 4.1. There are many ways to extend the free Schrödinger equation, i.e.

$$
\frac{\mathrm{d}}{\mathrm{d} t} u=-\mathrm{i} \Delta u
$$

into a time fractional equation (see for instance [Nab04], [Las02], [Luc13]). First, we may consider

$$
\mathbf{D}_{0, t}^{\alpha} u=-\mathrm{i} \Delta u
$$

However, as pointed out in [ER18], that equation has regularizing effect and dissipative properties, in a great contrast with (4.2). In order to recover a hyperbolic behavior, it is better to extend (4.2) by

$$
\mathbf{D}_{0, t}^{\alpha} u=-\mathrm{i}^{\alpha} \Delta u \text {. }
$$

Indeed, according to [ER18, Theorem 4.4 \& Example 4.6], this equation has no regularizing effect and possesses an asymptotic conservation law. The drawback of (4.3) regarding the dynamics, is that it does not generate a semi-group. However, (4.1) does, as we will show in the sequel.

Remark 4.2. The equation of (4.1) holds equivalently in $C_{\mathrm{b}}\left(\mathbb{R}, L^{2}\left(\mathbb{R}^{d}\right)\right)$. In any case, $\mathbf{D}_{-\infty, t}^{\alpha} u$ is understood in the sense of Definition 2.4.

Theorem 4.1. Let $\alpha \in(0,1), A: D(A) \subseteq L^{2}\left(\mathbb{R}^{d}\right) \rightarrow L^{2}\left(\mathbb{R}^{d}\right)$ be a positive self adjoint operator on $L^{2}\left(\mathbb{R}^{d}\right)$ and $v \in D(A)$. Then (4.1) has a unique solution $u$. Moreover, for all $t \in \mathbb{R}$,

$$
u(t)=\mathrm{e}^{\mathrm{i} t A^{1 / \alpha}} v, \quad \text { in } \quad D(A) .
$$

Before to prove this theorem, let us precise the meaning of (4.4). According to the Spectral Theorem in multiplication form (see [RS80, Theorem VIII.4] or [Hal13, Theorem 10.10]), there exist a measure space $(X, \mu)$ with finite measure $\mu$, a unitary map $\mathcal{U}: L^{2}\left(\mathbb{R}^{d}\right) \rightarrow L^{2}(X, \mu)$ and a measurable real-valued function $h$ on $X$ which is finite $\mu$-a.e., such that

$$
\mathcal{U}(D(A))=\left\{\psi \in L^{2}(X, \mu) \mid h \psi \in L^{2}(X, \mu)\right\}
$$

and

$$
\mathcal{U} A \mathcal{U}^{-1}(\psi)=h \psi, \quad \forall \psi \in \mathcal{U}(D(A)) .
$$

Under the assumption of Theorem 4.1, we claim that

$$
h \geq 0 \quad \mu \text {-a.e. on } X \text {. }
$$


Indeed, let us denote by $(\cdot, \cdot)_{L^{2}(X, \mu)}$ the standard inner product of $L^{2}(X, \mu)$. We put, for each positive integer $n$,

$$
X_{n}:=\{\xi \in X \mid-n<h(\xi)<0\},
$$

and denote by $\mathbb{I}_{X_{n}}$ the indicator function of $X_{n}$ (i.e. $\mathbb{I}_{X_{n}}=1$ on $X_{n}$ and 0 elsewhere). Then $h \mathbb{I}_{X_{n}}$ lies in $L^{2}(X, \mu)$ since $\mu$ is finite. Hence

$$
\begin{aligned}
\left(h \mathbb{I}_{X_{n}}, \mathbb{I}_{X_{n}}\right)_{L^{2}(X, \mu)} & =\left(\mathcal{U} A \mathcal{U}^{-1}\left(\mathbb{I}_{X_{n}}\right), \mathbb{1}_{X_{n}}\right)_{L^{2}(X, \mu)} & & (\text { by }(4.6)) \\
& =\left(A \mathcal{U}^{-1}\left(\mathbb{I}_{X_{n}}\right), \mathcal{U}^{-1}\left(\mathbb{I}_{X_{n}}\right)\right)_{L^{2}\left(\mathbb{R}^{d}\right)} & & \text { (since } \mathcal{U} \text { is unitary) } \\
& \geq 0 & & \text { (since } A \text { is positive) }
\end{aligned}
$$

Thus, $\mu\left(X_{n}\right)=0$; and since $h$ is finite $\mu$-a.e., we obtain (4.7).

We are now in position to define the operator $\mathrm{e}^{\mathrm{i} t A^{1 / \alpha}}$. For each $t \in \mathbb{R}$, denote by $f_{t}$, the function

$$
f_{t}:[0, \infty) \rightarrow \mathbb{C}, \quad x \mapsto \mathrm{e}^{\mathrm{i} t x^{1 / \alpha}} .
$$

Then $\mathrm{e}^{\mathrm{i} t A^{1 / \alpha}}$ is defined through bounded functional calculus for self adjoint operators (see [RS80, Theorem VIII.5] or [Kow09, Corollary 4.43]), by

$$
\mathrm{e}^{\mathrm{i} t A^{1 / \alpha}}:=f_{t}(A):=\mathcal{U}^{-1} M_{f_{t} \circ h} \mathcal{U},
$$

where $M_{f_{t} \circ h}: L^{2}(X, \mu) \rightarrow L^{2}(X, \mu)$ is the multiplication operator defined by

$$
M_{f_{t} \circ h} \psi=f_{t}(h) \psi, \quad \forall \psi \in L^{2}(X, \mu) .
$$

Proof of Theorem 4.1. (i) Uniqueness of the solution $u$. By linearity, it is enough to show that $v=0$ in (4.1) implies $u=0$. For each $\varphi \in \mathcal{S}(\mathbb{R})$, one has

$$
\begin{array}{rlrl}
\mathcal{U}\left(\left\langle\mathbf{D}_{-\infty, t}^{\alpha} u, \varphi\right\rangle_{\mathcal{S}^{\prime}\left(\mathbb{R} ; L^{2}\left(\mathbb{R}^{d}\right)\right)}\right) & =-\mathcal{U}\left(\int_{\mathbb{R}} u(t) \mathbf{D}_{t, \infty}^{\alpha} \varphi(t) \mathrm{d} t\right) & & \text { (by Definition 2.4) } \\
& =-\int_{\mathbb{R}} \mathcal{U}(u(t)) \mathbf{D}_{t, \infty}^{\alpha} \varphi(t) \mathrm{d} t & & \text { (by [ABHN11, Prop. 1.1.6]) } \\
& =\left\langle\mathbf{D}_{-\infty, t}^{\alpha} \hat{u}, \varphi\right\rangle_{\mathcal{S}^{\prime}\left(\mathbb{R} ; L^{2}(X, \mu)\right)} & \text { in } L^{2}(X, \mu),
\end{array}
$$

according to Definition 2.4 and with the notation $\hat{u}(t):=\mathcal{U}(u(t))$.

On the other hand,

$$
\begin{aligned}
\mathcal{U}\left(\left\langle\mathrm{i}^{\alpha} A u, \varphi\right\rangle_{\mathcal{S}^{\prime}\left(\mathbb{R} ; L^{2}\left(\mathbb{R}^{d}\right)\right)}\right) & =\mathcal{U}\left(\int_{\mathbb{R}} \mathrm{i}^{\alpha} A u(t) \varphi(t) \mathrm{d} t\right) \\
& \left.=\int_{\mathbb{R}} \mathrm{i}^{\alpha} h \hat{u}(t) \varphi(t) \mathrm{d} t \quad \text { (since } \mathcal{U} A(u(t))=h \hat{u}(t) \text { by }(4.6)\right) \\
& =\left\langle\mathrm{i}^{\alpha} h \hat{u}, \varphi\right\rangle_{\mathcal{S}^{\prime}\left(\mathbb{R} ; L^{2}(X, \mu)\right) .}
\end{aligned}
$$

Since $u$ solves (4.1), we get

$$
\mathbf{D}_{-\infty, t}^{\alpha} \hat{u}=\mathrm{i}^{\alpha} h \hat{u}, \quad \text { in } \mathcal{S}^{\prime}\left(\mathbb{R} ; L^{2}(X, \mu)\right) .
$$

In particular, for $\mu$-a.e. $\xi \in X$, there holds $h(\xi) \geq 0$ and

$$
\mathbf{D}_{-\infty, t}^{\alpha} \hat{u}(\cdot, \xi)=\mathrm{i}^{\alpha} h(\xi) \hat{u}(\cdot, \xi), \quad \text { in } \mathcal{S}^{\prime}(\mathbb{R}) .
$$

Besides, $\hat{u}(0, \xi)=\mathcal{U}(u(0))(\xi)=0$ for $\mu$-a.e. $\xi$ in $X$. Thus Theorem 3.1 leads to $\hat{u}(\cdot, \xi)=0$; so that $u=0$. That completes the uniqueness part of the proof. 
(ii) Existence. Let us show that the function

$$
u: t \mapsto \mathrm{e}^{\mathrm{i} t A^{1 / \alpha}} v
$$

solves (4.1). For, let $t \in \mathbb{R}$. With the notation $\hat{w}:=\mathcal{U}(w)$ for $w \in L^{2}\left(\mathbb{R}^{d}\right)$, we get in view of (4.8)

$$
\hat{u}(t)=f_{t}(h) \hat{v}
$$

Since $f_{t}$ is bounded $\mu$-a.e. on $X$, we deduce that $\hat{u}$ belongs to $L^{\infty}\left(\mathbb{R} ; L^{2}(X, \mu)\right)$. Then, thanks to Definition 2.4, we infer

$$
\left\langle\mathbf{D}_{-\infty, t}^{\alpha} \hat{u}, \varphi\right\rangle_{\mathcal{S}^{\prime}\left(\mathbb{R} ; L^{2}(X, \mu)\right)}=-\int_{\mathbb{R}} \hat{u}(t) \mathbf{D}_{t, \infty}^{\alpha} \varphi(t) \mathrm{d} t, \quad \text { in } L^{2}(X, \mu) .
$$

Thus for $\mu$-a.e. $\xi$ in $X$,

$$
\begin{array}{rlrl}
\left\langle\mathbf{D}_{-\infty, t}^{\alpha} \hat{u}, \varphi\right\rangle_{\mathcal{S}^{\prime}\left(\mathbb{R} ; L^{2}(X, \mu)\right)}(\xi) & =-\int_{\mathbb{R}} f_{t}(h(\xi)) \hat{v}(\xi) \mathbf{D}_{t, \infty}^{\alpha} \varphi(t) \mathrm{d} t \quad & & (\text { by }(4.10)) \\
& =\left\langle\mathbf{D}_{-\infty, t}^{\alpha}\left\{f_{t}(h(\xi)) \hat{v}(\xi)\right\}, \varphi\right\rangle_{\mathcal{S}^{\prime}(\mathbb{R})} & & \\
& =\left\langle i^{\alpha} h(\xi) \hat{u}(\cdot, \xi), \varphi\right\rangle_{\mathcal{S}^{\prime}(\mathbb{R})} \quad \text { (by Th. 3.1) }
\end{array}
$$

Hence we have proved that, for $\mu$-a.e. $\xi$ in $X$,

$$
\mathbf{D}_{-\infty, t}^{\alpha} \hat{u}(\cdot, \xi)=\mathrm{i}^{\alpha} h(\xi) \hat{u}(\cdot, \xi), \quad \text { in } S^{\prime}(\mathbb{R}) .
$$

Moreover, by (4.10),

$$
\|h \hat{u}(t, \cdot)\|_{L^{2}(X, \mu)}^{2}=\int_{X} h^{2}|\hat{v}|^{2} \mathrm{~d} \mu<\infty
$$

since $\hat{v}$ lies in $\mathcal{U}(D(A))$ - see (4.5). Hence $t \mapsto h \hat{u}(t)$ belongs to $L^{\infty}\left(\mathbb{R}, L^{2}(X, \mu)\right)$; so that, with (4.11), we derive that

$$
\mathbf{D}_{-\infty, t}^{\alpha} \hat{u}=\mathrm{i}^{\alpha} h \hat{u}, \quad \text { in } S^{\prime}\left(\mathbb{R}, L^{2}(X, \mu)\right) .
$$

Let us go back in the direct space $L^{2}\left(\mathbb{R}^{d}\right)$ and write down the expected equation for $u$. In view of (4.6), we have

$$
h \hat{u}(t)=\mathcal{U} A u(t), \quad \text { in } L^{2}(X, \mu) .
$$

Also (see above, the uniqueness part of the proof)

$$
\mathbf{D}_{-\infty, t}^{\alpha} \hat{u}=\mathcal{U}\left(\mathbf{D}_{-\infty, t}^{\alpha} u\right), \quad \text { in } \mathcal{S}^{\prime}\left(\mathbb{R}, L^{2}(X, \mu)\right) .
$$

Thus, with (4.12)

$$
\mathbf{D}_{-\infty, t}^{\alpha} u=\mathrm{i}^{\alpha} A u, \quad \text { in } \mathcal{S}^{\prime}\left(\mathbb{R} ; L^{2}\left(\mathbb{R}^{d}\right)\right) .
$$

Finally, $\hat{u}(0)=\hat{v}$, thus $u(0)=v$. Hence $u$ is solution to (4.1), which completes the proof of the theorem.

Theorem 4.1 allows us to define the solution operator associated to (4.1). Indeed, under the assumptions of that theorem, for each $t$ in $\mathbb{R}$, we put

$$
S_{\alpha}(t):=\mathrm{e}^{\mathrm{i} t A^{1 / \alpha}} .
$$

Then, for each $v$ in $D(A), t \mapsto S_{\alpha}(t) v$ is the solution to (4.1), according to Theorem 4.1. Thus, by definition, $S_{\alpha}$ is the solution operator associated to (4.1).

With these notation, we may give some properties of $S_{\alpha}$. 
Theorem 4.2. Let $\alpha \in(0,1)$ and $A$ be a positive self adjoint operator on $L^{2}\left(\mathbb{R}^{d}\right)$. Then $S_{\alpha}$ defined by (4.13), is a strongly continuous unitary group on $L^{2}\left(\mathbb{R}^{d}\right)$. Moreover, its infinitesimal generator is $\mathrm{i} A^{1 / \alpha}$.

Above, $A^{1 / \alpha}$ is defined through unbounded functional calculus. More precisely, denoting by $\mathcal{L}\left(L^{2}\left(\mathbb{R}^{d}\right)\right)$ the space of linear and continuous maps from $L^{2}\left(\mathbb{R}^{d}\right)$ into itself, Theorem 10.4 in [Hal13] states that there exists a unique projection-valued measure $\mu^{A}$ on $[0, \infty)$ with values in $\mathcal{L}\left(L^{2}\left(\mathbb{R}^{d}\right)\right)$ such that

$$
\int_{0}^{\infty} \lambda \mathrm{d} \mu^{A}=A
$$

Then

$$
A^{1 / \alpha}:=\int_{0}^{\infty} \lambda^{1 / \alpha} \mathrm{d} \mu^{A}
$$

The domain of $A^{1 / \alpha}$ is

$$
D\left(A^{1 / \alpha}\right):=\left\{v \in L^{2}\left(\mathbb{R}^{d}\right) \mid \int_{0}^{\infty} \lambda^{2 / \alpha} \mathrm{d} \mu_{v}^{A}(\lambda)<\infty\right\},
$$

where $\mu_{v}^{A}$ is the Borel measure on $[0, \infty)$ defined for each Borel set $E \subseteq[0, \infty)$ by

$$
\mu_{v}^{A}(E)=\left(v, \mu^{A}(E) v\right)_{L^{2}\left(\mathbb{R}^{d}\right)} .
$$

We recall that $\mu^{A}(E)$ is an orthogonal projection of $L^{2}\left(\mathbb{R}^{d}\right)$.

Proof of Theorem 4.2. Recalling that $f_{t}(x):=\mathrm{e}^{\mathrm{i} t x^{1 / \alpha}}$, it is clear from (4.8) that $S_{\alpha}(t)$ is bounded on $L^{2}\left(\mathbb{R}^{d}\right)$. Also, $S_{\alpha}(0)$ is equal to $I d_{L^{2}\left(\mathbb{R}^{d}\right)}$, the identity operator of $L^{2}\left(\mathbb{R}^{d}\right)$. Moreover, for each real numbers $t, s$, the properties of the functional calculus featured in [RS80, Theorem VIII.5] lead to

$$
S_{\alpha}(t) S_{\alpha}(s)=f_{t}(A) f_{s}(A)=f_{t+s}(A)=S_{\alpha}(t+s) .
$$

Let us show that $S_{\alpha}(t)$ is unitary. For, again by functional calculus, we have

$$
\begin{aligned}
S_{\alpha}(t) S_{\alpha}(t)^{*}=f_{t}(A) \overline{f_{t}}(A) & =\left|f_{t}\right|^{2}(A) \\
& =I d_{L^{2}\left(\mathbb{R}^{d}\right)}
\end{aligned}
$$

since $\left|f_{t}(x)\right|=1$ for $x \geq 0$. In the same way, there holds $S_{\alpha}(t)^{*} S_{\alpha}(t)=I d_{L^{2}\left(\mathbb{R}^{d}\right)}$, so that $S_{\alpha}(t)$ is unitary.

Furthermore, by (4.6) and (4.8), for each $v$ in $L^{2}\left(\mathbb{R}^{d}\right)$,

$$
\begin{aligned}
\left\|S_{\alpha}(t) v-v\right\|_{L^{2}\left(\mathbb{R}^{d}\right)}^{2} & =\left\|M_{f_{t} \circ h} \hat{v}-\hat{v}\right\|_{L^{2}(X, \mu)}^{2} \\
& =\int_{X}\left|\mathrm{e}^{\mathrm{i} t h(\xi)^{1 / \alpha}}-1\right|^{2}|\hat{v}(\xi)|^{2} \mathrm{~d} \mu(\xi) \underset{t \rightarrow 0}{\longrightarrow} 0,
\end{aligned}
$$

by the Lebesgue dominated convergence theorem. Hence, $S_{\alpha}$ is strongly continuous.

Finally, let us compute its infinitesimal generator. According to [RS80, Theorem VIII.6], there holds

$$
S_{\alpha}(t)=\int_{0}^{\infty} f_{t}(\lambda) \mathrm{d} \mu^{A}
$$

Hence, for each $v$ in $D\left(A^{1 / \alpha}\right)$, Theorem 5.9 in [Sch12, Chap 5] leads to

$$
\left\|\frac{S_{\alpha}(t) v-v}{t}-\mathrm{i} A^{1 / \alpha} v\right\|_{L^{2}\left(\mathbb{R}^{d}\right)}^{2}=\int_{0}^{\infty}\left|\frac{\mathrm{e}^{\mathrm{i} t \lambda^{1 / \alpha}}-1}{t}-\mathrm{i} \lambda^{1 / \alpha}\right|^{2} \mathrm{~d} \mu_{v}^{A}(\lambda) .
$$


Using the fact that $\lambda \mapsto \lambda^{2 / \alpha}$ lies in $L^{1}\left(0, \infty ; \mu_{v}^{A}\right)$ (by (4.14)), we obtain with the dominated convergence theorem, that the latter integral goes to zero as $t \rightarrow 0$. That is to say i $A^{1 / \alpha}$ is the infinitesimal generator of $S_{\alpha}$.

The next result states that the time fractional problem (4.1) may be reduced to a time first order PDE. Notice that the latter PDE is obtained formally by raising the differential operators involved in (4.1) to the power $1 / \alpha$.

Corollary 4.3. Let $\alpha \in(0,1)$, A be a positive self adjoint operator on $L^{2}\left(\mathbb{R}^{d}\right)$ and $v \in D\left(A^{1 / \alpha}\right)$. Then (4.1) is equivalent to the following problem

$$
\left\{\begin{array}{l}
\text { Find } u \in C^{1}\left(\mathbb{R}, L^{2}\left(\mathbb{R}^{d}\right)\right) \cap C\left(\mathbb{R}, D\left(A^{1 / \alpha}\right)\right) \text { such that } \\
\frac{\mathrm{d}}{\mathrm{d} t} u=\mathrm{i} A^{1 / \alpha} u, \quad \text { in } C\left(\mathbb{R}, L^{2}\left(\mathbb{R}^{d}\right)\right) \\
u(0)=v .
\end{array}\right.
$$

Proof. If $u$ solves $(4.1)$ then $u(t)=\exp \left(\mathrm{it} A^{1 / \alpha}\right) v$ by Theorem 4.1. Then $u$ is solution to (4.16) according to Theorem 4.2. Moreover, functional calculus tells us that $A^{1 / \alpha}$ is self adjoint on $L^{2}\left(\mathbb{R}^{d}\right)$. Thus (see for instance [Sch12, Proposition 6.5]), (4.16) has a unique solution. Consequently, (4.1) and (4.16) are equivalent.

Finally, as an example, let us consider the case

$$
A:=-\Delta+V
$$

where $V \in L^{\infty}\left(\mathbb{R}^{d}\right)$ is non negative a.e. on $\mathbb{R}^{d}$, and $D(A)=H^{2}\left(\mathbb{R}^{d}\right)$. Then, for $\alpha \in(0,1)$ and $v$ in $H^{2}\left(\mathbb{R}^{d}\right)$, the time fractional Schrödinger problem

$$
\mathbf{D}_{-\infty, t}^{\alpha} u=\mathrm{i}^{\alpha}(-\Delta+V) u, \quad u(0)=v,
$$

has a unique solution $u$, according to Theorem 4.1. Moreover,

$$
u(t)=S_{\alpha}(t) v=\mathrm{e}^{\mathrm{i} t(-\Delta+V)^{1 / \alpha}} v, \quad \forall t \in \mathbb{R} .
$$

The requirements given in the introduction are satisfied, namely (i) the $L^{2}$-norm of $u(t)$ is conserved, i.e.

$$
\|u(t)\|_{L^{2}\left(\mathbb{R}^{d}\right)}=\|v\|_{L^{2}\left(\mathbb{R}^{d}\right)}, \quad \forall t \in \mathbb{R}
$$

and (ii) $S_{\alpha}$ is a group, i.e.

$$
S_{\alpha}(t) S_{\alpha}(s)=S_{\alpha}(t+s), \quad \forall t, s \in \mathbb{R} .
$$

Also, if $v$ belongs to the domain of $(-\Delta+V)^{1 / \alpha}$ then Corollary 4.3 yields that $u$ given by (4.17) is the unique solution to

$$
\frac{\mathrm{d}}{\mathrm{d} t} u=\mathrm{i}(-\Delta+V)^{1 / \alpha} u, \quad u(0)=v .
$$

\section{REFERENCES}

[ABHN11] Wolfgang Arendt, Charles J. K. Batty, Matthias Hieber, and Frank Neubrander. Vector-valued Laplace transforms and Cauchy problems, volume 96 of Monographs in Mathematics. Birkhäuser/Springer, Basel, second edition, 2011.

[BMRST16] Ana Bernardis, Francisco J. Martín-Reyes, Pablo Raúl Stinga, and José L. Torrea. Maximum principles, extension problem and inversion for nonlocal one-sided equations. J. Differential Equations, 260(7):6333-6362, 2016. 
[Die10] Kai Diethelm. The analysis of fractional differential equations, volume 2004 of Lecture Notes in Mathematics. Springer-Verlag, Berlin, 2010.

[ER17] Hassan Emamirad and Arnaud Rougirel. Solution operators of three time variables for fractional linear problems. Mathematical Methods in the Applied Sciences, 40(5):1553$1572,2017$.

[ER18] Hassan Emamirad and Arnaud Rougirel. Time fractional linear problems on $L^{2}\left(\mathbb{R}^{d}\right)$. Bull. Sci. Math., 144:1-38, 2018.

[Hal13] Brian C. Hall. Quantum theory for mathematicians, volume 267 of Graduate Texts in Mathematics. Springer, New York, 2013.

[Kow09] Marko Kowalski. Spectral Theory in Hilbert Spaces. http://www.math.ethz.ch/吕owalski/spectral-theory.pdf, 2009.

[Las02] Nick Laskin. Fractional Schrödinger equation. Phys. Rev. E (3), 66(5):056108, 7, 2002.

[Lov38] E. R. Love. Fractional Integration, and Almost Periodic Functions. Proc. London Math. Soc. (2), 44(5):363-397, 1938.

[Luc13] Yuri Luchko. Fractional Schrödinger equation for a particle moving in a potential well. J. Math. Phys., 54(1):012111, 10, 2013.

[Nab04] Mark Naber. Time fractional Schrödinger equation. J. Math. Phys., 45(8):3339-3352, 2004.

[RS80] Michael Reed and Barry Simon. Methods of modern mathematical physics. I. Academic Press, Inc., New York, second edition, 1980. Functional analysis.

[Sch12] Konrad Schmüdgen. Unbounded self-adjoint operators on Hilbert space, volume 265 of Graduate Texts in Mathematics. Springer, 2012.

[SKM93] Stefan G. Samko, Anatoly A. Kilbas, and Oleg I. Marichev. Fractional integrals and derivatives. Gordon and Breach Science Publishers, Yverdon, 1993.

HASSAN EMAMIRAD

School of Mathematics, Institute for Research in Fundamental Sciences (IPM), P.O. Box 19395-5746, TEHRAN, Iran

Laboratoire de Mathématiques, Université de Poitiers \& CNRS. Téléport 2, BP 179, 86960 Chassneuil du Poitou Cedex, France

E-mail address: emamirad@ipm.ir

E-mail address: emamirad@math.univ-poitiers.fr

Arnaud Rougirel

Laboratoire de Mathématiques, Université de Poitiers \& CNRS. Téléport 2, BP 179, 86960 Chassneuil du Poitou Cedex, France

E-mail address: rougirel@math.univ-poitiers.fr 